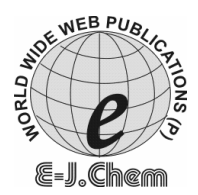

http://www.e-journals.net
ISSN: 0973-4945; CODEN ECJHAO

E-Journal of Chemistry

Vol. 5, No.4, pp. 718-722, October 2008

\title{
A Facile Conversion of Alcohols to Esters Mediated by Potassium Ferrate
}

\author{
M. KOOTI*, A. TARASSOLI, H. JAVADI and M. JORFI \\ Department of Chemistry, College of Science, \\ Shahid Chamran University, Ahvaz, Iran. \\ m_kooti@scu.ac.ir
}

Received 23 December 2007; Accepted 20 February 2008

\begin{abstract}
Potassium ferrate in the presence of copper(II) sulfate pentahydrate can convert 1-phenyl ethanol, 4-chloro-1-phenyl ethanol and 2-phenyl ethanol into ester products with good or excellent yields. The reactions have been carried out in $n$-hexane at room temprature.The effect of other metal salts, as activators, have been also examined instead of copper sulfate.
\end{abstract}

Keywords: Potassium ferrate, 1-Phenyl ethanol, 2-Phenyl ethanol, Copper(II) sulfate, Ester.

\section{Introduction}

Several high-valent transition metal oxyanions have been widely used in organic synthesis as oxidants. However, with the increasing concerns over toxic effects of such species, the development of less polluting oxidants has become a priority for chemists. Potassium ferrate $\left(\mathrm{FeO}_{4}{ }^{2-}\right)$ can be considered as an alternative green oxidant since it produces iron oxides in the oxidation reactions ${ }^{1,3}$. Although ferrate is thermodynamically stronger oxidant than $\mathrm{MnO}_{4}^{-}$and $\mathrm{Cr}_{2} \mathrm{O}_{7}{ }^{2-}$, but unlike these oxidants, it is not indiscriminate reagent and shows no reactivity toward alkene and alkyne functionalities. There are some reports, however, of using ferrate for oxidation of alcohols and amine $e^{4,5}$. The study by Delaude and Laszlo ${ }^{2}$ showed that ferrate combined with suitable heterogeneous catalyst (e.g. K10 clay) could oxidize the functional groups of organic compounds with product yields. Other researchers reported that hydrated metal salts can activate permanganate and ferrate in some oxidation processes but no comment was made on the role of these salts ${ }^{6,7}$. To extend this line of research and gaining an insight into the role of metal salts in these processes, we have examined the oxidation of a few alcohols by ferrate in the presence of various hydrated metal salts. To our surprise, we have observed that the oxidation of 1-phenyl ethanol, 4-chloro-1-phenyl ethanol and 2-phenyl ethanol by potassium ferrate in the presence of copper(II) sulfate pentahydrate led to the production of ester products. 


\section{Experimental}

All reactions were carried out at room temperature using $n$-hexane as solvent. The reported yields refer to isolated products which were characterized by comparison of their physical and spectral data with those of authentic samples. Copper(II) sulfate or other metal salts were finely powdered before use.

\section{General oxidation reaction}

A mixture of potassium ferrate $(0.3 \mathrm{~g}, 1.5 \mathrm{mmol})$, copper(II) sulfate pentahydrate $(0.25 \mathrm{~g}$ $1.0 \mathrm{mmol})$ and 1-phenyl ethanol $(0.061 \mathrm{~g} 0.5 \mathrm{mmol})$ was suspended in $30 \mathrm{~mL}$ of $n$-hexane and stirred at room temperature for $24 \mathrm{~h}$. The progress of the reaction was monitored by TLC using ethyl acetate- $\mathrm{CCl}_{4}$. The mixture was then filtered off and the filter cake was washed with dichloromethane $(10 \mathrm{~mL})$. The solvent was removed under reduced pressure to afford $70 \%$ yield of methyl benzoate ester. Other reactions have been carried out in the same manner using 3:2:1 molar ratio of potassium ferrate, copper sulfate and alcohol respectively. In all cases the yields refers to isolated products and the products were characterized by spectroscopic measurements.

\section{Results and Discussions}

We have used the method introduced by Delaude ${ }^{2}$ for the preparation of potassium ferrate and carried out the oxidation reactions in $n$-hexane. Thus, a mixture of alcohol, potassium ferrate and copper(II) sulfate pentahydrate, or other metal salts, in a molar ratio of 1:3:2 respectively, was suspended in $n$-hexane. The suspension was stirred at room temperature and the reaction was monitored by TLC. The final isolated products were characterized by IR and ${ }^{1} \mathrm{HNMR}$ and ${ }^{13} \mathrm{CNMR}$ spectroscopy and by direct comparison with authentic samples. 1-Phenyl ethanol was selected as probe substrate in our investigations.

As expected, the oxidation of 1-phenyl ethanol by ferrate catalyzed with copper sulfate gave acetophenone at first. But, interestingly, upon stirring for a longer time to get more of the ketone product, we noticed that the ketone started converting to a new product identified as methyl benzoate (Scheme 1).

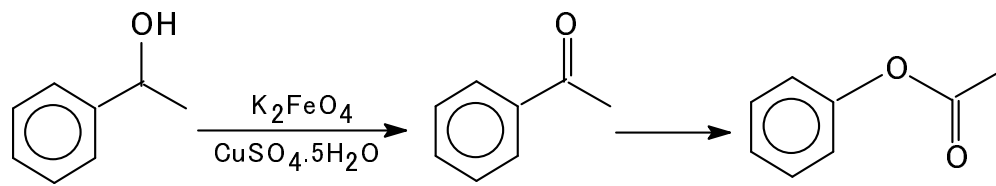

Scheme 1

The yield of this ester was $70 \%$ after $24 \mathrm{~h}$ of reaction time. To our knowledge, there is no report of similar one-pot conversion of alcohol to ester mediated by ferrate or other oxyanions in such mild conditions. Thus, we have performed some experiments to find out the effects of various factors on the outcome of this reaction.

First, we have examined the effect of the moisture content of copper(II) sulfate on the reaction by carrying out the oxidation of 1-phenyl ethanol with $\mathrm{K}_{2} \mathrm{FeO}_{4}$ using thoroughly dried copper sulfate. We found that this alcohol resisted oxidation to ketone in this condition. This reveals that the presence of trace quantities of water is crucial for the reaction to proceed. The lack of reactivity in this case might be due to the absorption of the alcohol by anhydrous salt and therefore the alcohol will not be free to interact with potassium ferrate. Our finding is in consistent with the results of other reported works ${ }^{2,9}$, 
The catalytic activity of hydrated copper(II) sulfate is not fully understood at present. It may act as a source of moisture and/or participate in electron transfer. We, therefore, have carried out a series of experiments using other hydrated metal salts instead of copper(II) sulfate. These include, $\mathrm{CuCl}_{2} \cdot 2 \mathrm{H}_{2} \mathrm{O}, \mathrm{Cu}\left(\mathrm{NO}_{3}\right)_{2} \cdot 3 \mathrm{H}_{2} \mathrm{O}, \mathrm{NiSO}_{4} \cdot 6 \mathrm{H}_{2} \mathrm{O}, \mathrm{MgCl}_{2} \cdot 6 \mathrm{H}_{2} \mathrm{O}$, $\mathrm{MgSO}_{4} .7 \mathrm{H}_{2} \mathrm{O}$ and $\mathrm{ZnSO}_{4} \cdot 7 \mathrm{H}_{2} \mathrm{O}$. Interestingly, no one of the mentioned salts exhibited a better activating effect than copper sulfate. That is, oxidation of 1-phenyl ethanol by ferrate in the presence of each of these salts resulted only to a small amount of acetophenone and no ester product was observed. This observation can be tentatively explained by assuming that the activation effect of metal salt is due to the coordination of ferrate moiety to the metal centre through an oxo ligand. This is most likely similar to the protonation of $\mathrm{FeO}_{4}{ }^{2-}$, and the metal acts as Lewis acid forming adduct with ferrate as shown below.

$$
\left[\mathrm{FeO}_{4}\right]^{2-}+\mathrm{M}^{2+} \rightarrow\left[\mathrm{O}_{3} \mathrm{Fe}=\mathrm{O} \rightarrow \mathrm{M}\right]
$$

The protonated ferrate species is thermodynamically a much stronger oxidant, and similarly the withdrawing of electron density by the metal in the formed adduct causes ferrate ion to be more oxidizing. In view of our results, it might be reasonable to indicate that, except copper sulfate, all of the tested metal salts were not capable of forming a strong coordinate bond with ferrate. Some of these salts, including nickel and magnesium salts lack the vacant sites for coordination, copper chloride and nitrate, in the other hand, are weak Lewis acids ${ }^{10}$. The zinc ion in zinc sulfate is considered as a soft acid which can not form stable adduct with the hard oxo ligand of ferrate. The best catalyst for activating ferrate seemed to be copper(II) sulfate. In this salt, two sulfate groups are coordinated to the metal and the high electronegativity of oxygen atoms of the sulfate ligands increases the Lewis acidity of the central metal. This will lead to the formation of strong oxo-metal bond and bring about a stable ferrate adduct, which in turn enhancing the oxidizing power of the ferrate ion.

In another experiment, we have examined the influence of the temperature on the rate and the yield of the reaction. Thus, the oxidation of 1-phenyl ethanol by ferrate in the presence of copper(II) sulfate was performed at $50^{\circ} \mathrm{C}$ and reflux conditions but the afforded yields were disappointing. Although the conversion of ketone to ester in these conditions took place faster than at room temperature but the obtained yields of esters were rather poor. This might be due to the decomposition of ferrate ion at higher temperatures.

As we have mentioned, the oxidation of 1- phenyl ethanol by $\mathrm{FeO}_{4}{ }^{2-}$ produces acetophenone first and then this ketone, in somehow, transformed to ester. Therefore, we anticipated that reacting of acetophenone with $\mathrm{K}_{2} \mathrm{FeO}_{4}$ in the presence of copper sulfate will lead to the production of ester. However, this was not the case and this reaction afforded only a small yield of ester product even after prolonged reaction time $(24 \mathrm{~h})$. This result indicates that a good yield of ester product can be only generated during the course of the oxidation of the 1-phenyl ethanol.

In the same manner, we have examined the oxidation of 4-chloro-1-phenyl ethanol by ferrate using copper sulfate as activator. This alcohol was also successfully converted to its related ester product in $75 \%$ yield after $5 \mathrm{~h}$ reaction time. However, the oxidation of 2-phenyl ethanol occurred only on addition of alumina $(5 \mathrm{~g})$ to the reaction mixture. Thus, the reaction of this alcohol with ferrate in the presence of copper sulfate and alumina resulted to $95 \%$ yield of methyl benzoate after $16 \mathrm{~h}$ of reaction time. Surprisingly, both1-phenyl ethanol and 2-phenyl ethanol were converted to the same ester (methyl benzoate) according to their FTIR and NMR spectra measurements. Moreover, changing some factors in the oxidation reaction of 2-phenyl ethanol, such as, increasing the temperature or using dried copper sulfate did not bring about any improvement in the rate of the reaction, as seen for 1-phenyl ethanol oxidation (Table 1). 
Table1. Oxidation of some alcohols to esters in different condition

\begin{tabular}{|c|c|c|c|c|}
\hline Entry & Substrate & Product & Time, $\mathrm{h}$ & Yield, $\%^{\mathrm{a}}$ \\
\hline 1 & 1-Phenyl ethanol & Methyl benzoate & 24 & 70 \\
\hline 2 & 1-Phenyl ethanol ${ }^{\mathrm{b}}$ & Methyl benzoate & 5 & 0 \\
\hline 3 & 1-Phenyl ethanol ${ }^{\mathrm{c}}$ & Methyl benzoate & 24 & 0 \\
\hline 4 & 4-Choloro 1-Phenyl ethanol & 4-Choloro methyl benzoate & 5 & 72 \\
\hline 5 & 2-Phenyl ethanol ${ }^{\mathrm{d}}$ & Methyl benzoate & 16 & 95 \\
\hline 6 & 1-Phenyl ethanol & Ethyl benzoate & 24 & Trace \\
\hline
\end{tabular}

${ }^{a}$ Isolated yields. ${ }^{b}$ Reflux condition. ${ }^{c}$ Dried copper sulfate ${ }^{d}$ In the presence of alumina

In addition to these three studied alcohols, we have also examined the oxidation of 1-phenyl propanol, indanol and benzyl alcohol by our oxidation system in the same manner. The oxidation of these alcohols, however, resulted only in the production of their corresponding carbonyl compounds and no ester product, which is of our interest, was obtained (Table 1).

We have no clear explanation for the transformation of these alcohols to esters by our oxidation procedure. Whether Baeyer-Villiger mechanism is involved in this reaction or not could be only resolved after further extensive study. However, the mechanism shown in the following scheme may be tentatively proposed for this conversion.

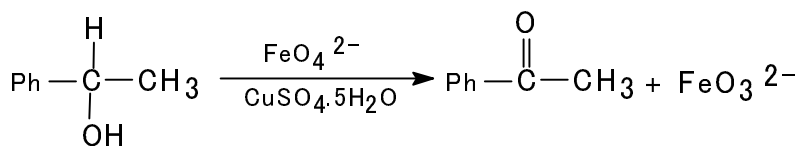

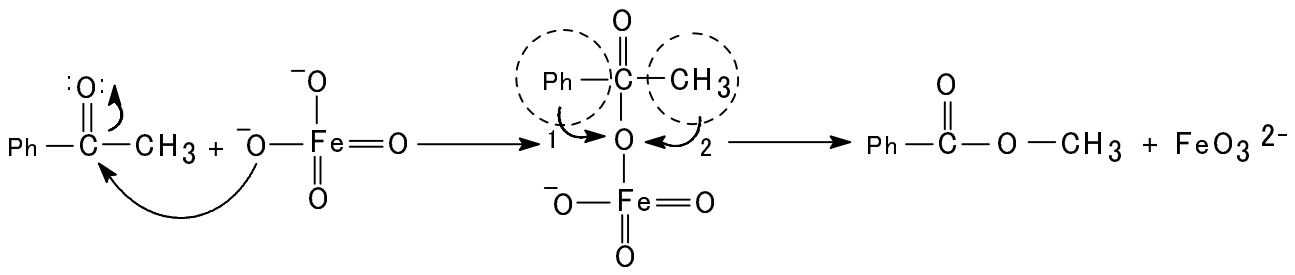

Scheme 2

The sequential steps of the suggested mechanism include the oxidation of 1-phenyl ethanol to acetophenone first and then this ketone forms adduct with ferrate ion through an oxo ligand to carbon atom of the carbonyl group. The electron withdrawing of $\mathrm{Fe}$ (VI) causes splitting of the Fe-O bond and transferring of oxygen atom to the ketone. The next step will be the migration of either phenyl (migration 1 in the scheme) or methyl (migration 2 in the scheme) group to the newly attached oxygen atom to produce the final ester. Our finding clearly showed that the only migrated group is the methyl one according to the FTIR and NMR spectra of the product.

In conclusion, the direct conversion of an alcohol to ester without the use of the corresponding acid is an attractive approach for generating esters. Although there are some reports of such transformations ${ }^{11,12}$, but the method we introduced may be considered as a facile, one-pot and simple rout for direct synthesis of at least some esters from alcohols. 


\section{Acknowledgement}

The authors appreciate the support offered by the Research Council of Shahid Chamran University, Ahvaz, Iran.

\section{References}

1. Lee D G and Gai A H, Can J Chem., 1993, 71, 1394.

2. Delaude L and Laszlo P, J Org Chem., 1996, 61, 6360.

3. Yunbo Lee, Min Cho and Jee Yeon Kim, J Ind Eng Chem., 2004, 10, 161.

4. Firouzabadi H and Ghaderi E, Tetrahedron Lett., 1978, 839.

5. Tsuda Y and Nakajima S, Chem Lett., 1978, 1397.

6. Norcross B E, Lewis W C, Gai A H and Lee N A D C, Can J Chem., 1997, 75, 129.

7. Kim K S, Song Y H, Lee N H, Halm C and Noureldin S, Tetrahedron Lett., 1986, 27, 2875.

8. $\quad$ Regen S L and Koteel C, J Am Chem Soc., 1977, 99, 3837.

9. Chi-Ming Ho and Tai-Chu Lau, New J Chem., 2000, 24, 587.

10. Wells A F, Structural Inorganic Chemistry, Oxford Clarenden Press, 1984.

11. Brooks E, Maki Audrey Chan and Eric M. Phillips, Org Lett., 2007, 9, 371.

12. Zhang J, Leitus G, Ben David Y and David M, J Am Chem Soc., 2005, 127, 10840. 


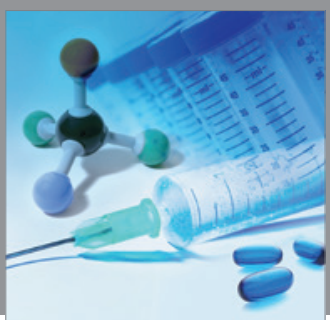

International Journal of

Medicinal Chemistry

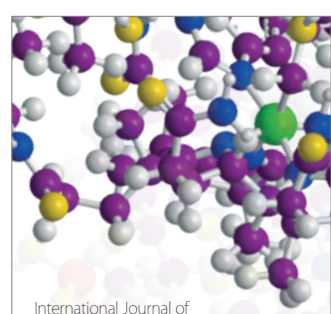

Carbohydrate Chemistry

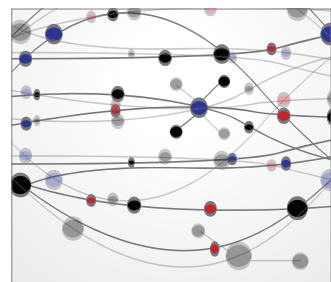

The Scientific World Journal
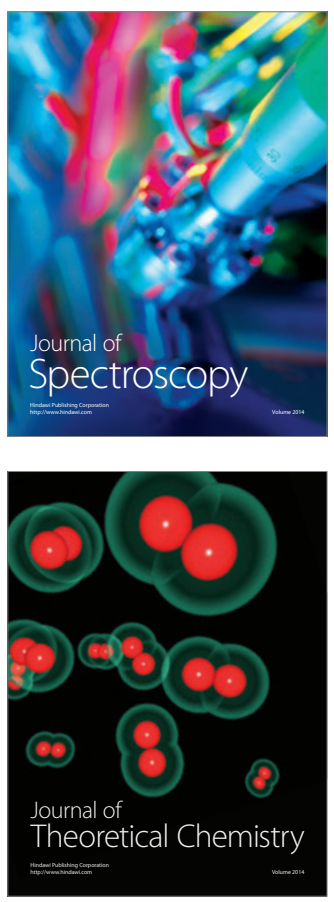
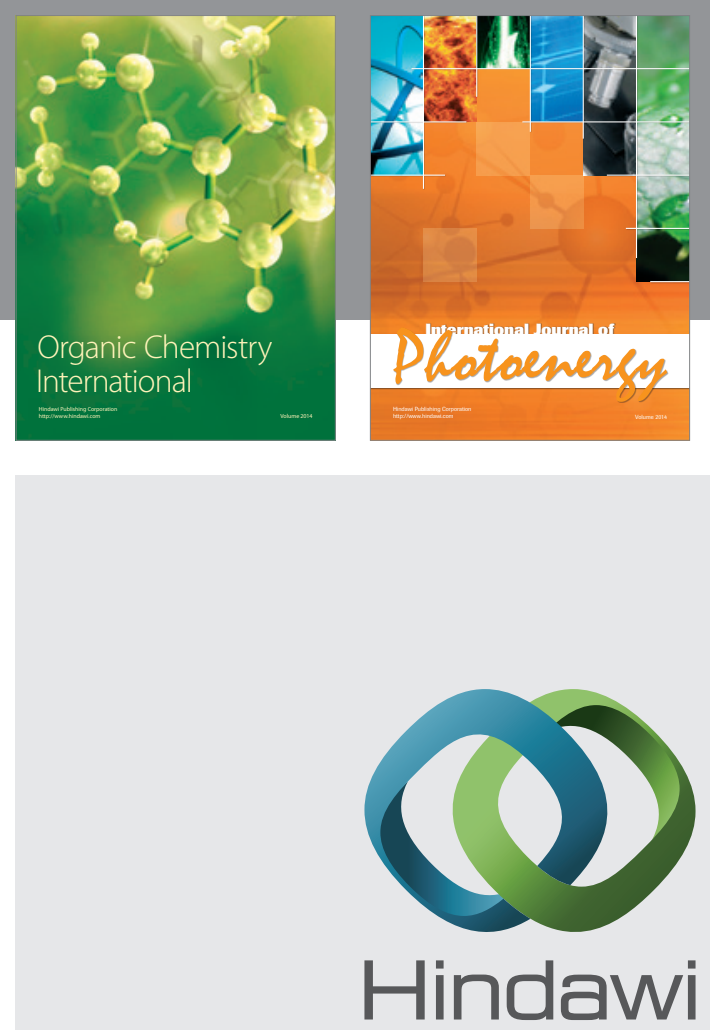

Submit your manuscripts at

http://www.hindawi.com
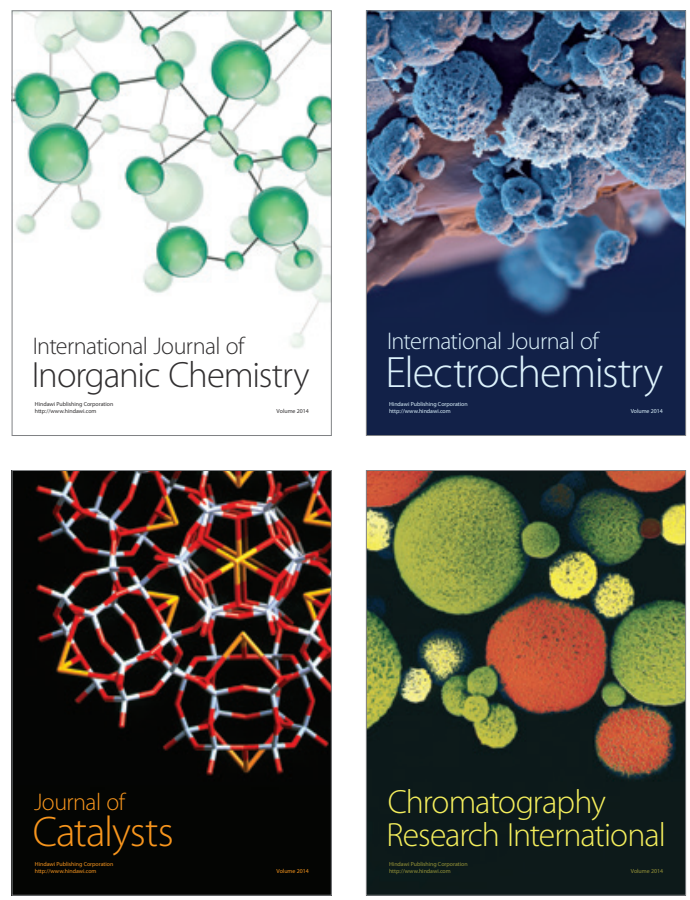
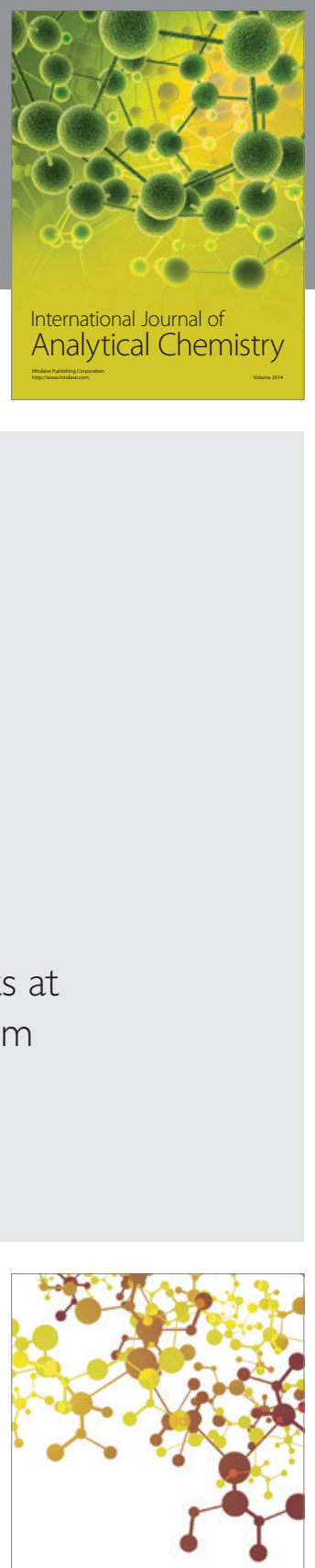

Journal of

Applied Chemistry
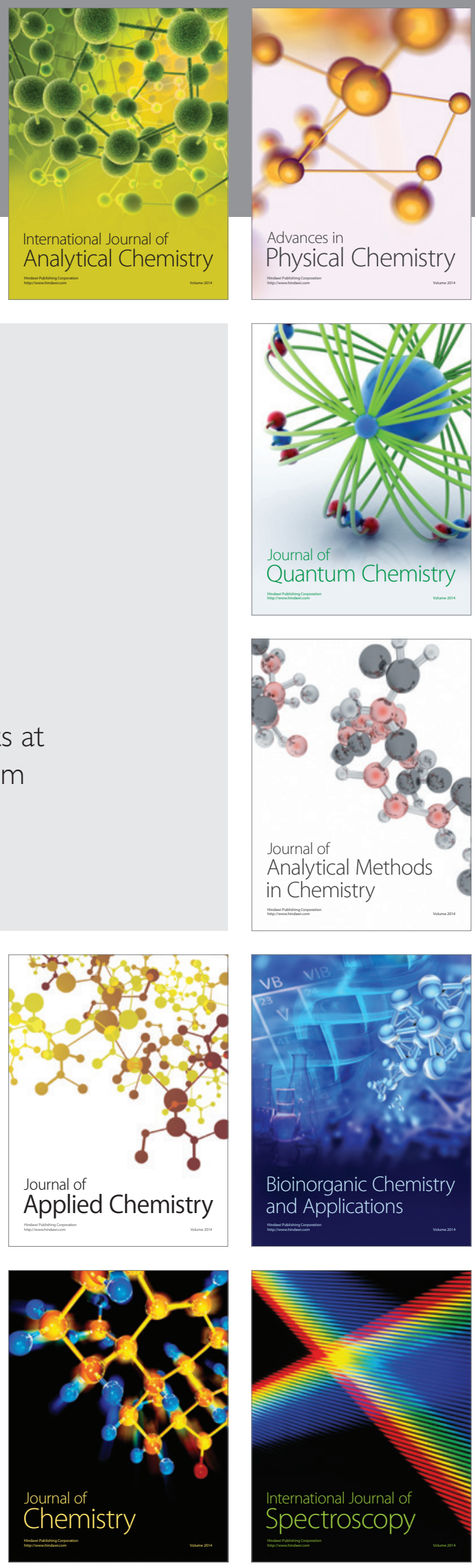